\title{
Differences in gut microbiota composition in metabolic syndrome and type 2 diabetes subjects in a multi-ethnic population: the HELIUS study
}

\author{
Mélanie Deschasaux $^{1}$, Kristien Bouter ${ }^{2}$, Andrei Prodan ${ }^{2}$, Evgeni Levin $^{2}$, Albert Groen ${ }^{2}$, \\ Hilde Herrema ${ }^{2}$, Valentina Tremaroli ${ }^{3}$, Marieke Snijder ${ }^{4}$, Mary Nicolaou ${ }^{4}$, Aeilko Zwinderman ${ }^{1}$, \\ Fredrik Bäckhed ${ }^{3}$ and Max Nieuwdorp ${ }^{2}$ \\ ${ }^{1}$ Department of Clinical Epidemiology and Biostatistics, Amsterdam University Medical Center, Amsterdam, \\ Netherlands, \\ ${ }^{2}$ Department of Internal and Vascular Medicine, Amsterdam University Medical Center, Amsterdam, Netherlands, \\ ${ }^{3}$ Wallenberg Laboratory, Sahlgrenska Hospital, University of Gothenburg, Gothenburg, Sweden and \\ ${ }^{4}$ Department of Public Health, Amsterdam University Medical Center, Amsterdam, Netherlands
}

\begin{abstract}
Recently, increased attention has been drawn to the composition of the intestinal microbiota and its possible role in metabolic syndrome and type 2 diabetes (T2DM). However, potential variation in gut microbiota composition across ethnic groups is rarely considered despite observed unequal prevalence for these diseases. Our objective was therefore to study the gut microbiota composition across health, metabolic syndrome and T2DM in a multi-ethnic population residing in the same geographical area. 16S rRNA gene sequencing was performed on fecal samples from 3926 participants to the HELIUS cohort (Amsterdam, The Netherlands), representing 6 ethnic groups (Dutch, Ghanaians, Moroccans, Turks, Surinamese of either African or South-Asian descent). Included participants completed a questionnaire and underwent a physical examination and overnight fasted blood sampling. Gut microbiota composition was compared across metabolic status (diabetes with and without metformin use, metabolic syndrome and its subsequent components, health) and ethnicities using Wilcoxon-Mann-Withney tests and logistic regressions. Overall, the gut microbiota alphadiversity (richness, Shannon index and phylogenetic diversity) decreased with worsening of the metabolic state (comparing health to metabolic syndrome to T2DM) but this was only partially reproduced in ethnic-specific analyses. In line, a lower alpha-diversity was found in relation to all metabolic syndrome components as well as in T2DM subjects using metformin compared to non-users. Alterations, mainly decreased abundances, were also observed at the genus level (many Clostridiales) in metabolic syndrome subjects and more strongly in T2DM subjects with differences across ethnic groups. In particular, we observed decreased abundances of members of the Peptostreptococcaceae family and of Turicibacter and an increased abundance of a member of the Enterobacteriaceae family. Our data highlight several compositional differences in the gut microbiota of individuals with metabolic syndrome or T2DM. These features, confirming prior observations, give some insights into potential key intestinal bacteria related to a worsening of metabolic state. Our results also underscore possible ethnic-specific profiles associated with these microbiota alterations that should be further explored.
\end{abstract}

\section{Conflict of Interest}

MN is in the Scientific Advisory Board of Caelus Pharmaceuticals, the Netherlands; FB is in the Scientific Advisory Board of MetaboGen AB, Sweden. None of these are directly relevant to the current paper. There are no patents, products in development or marketed products to declare. The other authors declare no conflict of interest 